\title{
MedienPädagogik
}

www. medienpaed.com

Zeitschrift für

Theorie und Praxis

der Medienbildung

ISSN 1424-3636

\section{Lernen mit Computer/Internet und Schulbuch Ein Prozessmodell zur theoriegeleiteten empirischen Erforschung der schulrelevanten Medienrezeption Jugendlicher in der Freizeit und ih- rer Auswirkungen auf den Kompetenzerwerb mit Medien in der Schule}

Stephan Kyas

\begin{abstract}
Im Beitrag werden aus Desideraten des aktuellen Forschungsstands zur bildungsorientierten Medienrezeption und zum Umgang mit Schulbüchern weitere Forschungsmöglichkeiten zum Lernen mit Computer/Internet und Schulbüchern abgeleitet. Darauf aufbauend wird ein Forschungskonzept vorgestellt, mit dem die informelle schulrelevante Medienrezeption mit Computer/Internet und Schulbüchern einschliesslich ihres Bedingungsgefüges sowie ihrer Effekte auf den formalen Kompetenzerwerb mit diesen Medien aus Schülersicht untersucht werden können. Das Konzept mündet in ein Prozessmodell, in dem unterschiedliche theoretische Ansätze triangulativ zusammengeführt und in ihrer spezifischen Bedeutung für ein mögliches empirisches Vorgehen skizziert werden.
\end{abstract}

\section{Einleitung: Medienrezeption und Kompetenzerwerb von Jugendlichen}

Zur alltäglichen Lebenswelt Jugendlicher gehört heute ein breites Repertoire an verfügbaren Medien, die vor allem ausserhalb der Schule rezipiert werden und die Konstruktion sozialer Wirklichkeit in bedeutsamer Weise beeinflussen können (Vollbrecht 2003). Computer und Internet bestimmen zu einem grossen Teil die mediale Freizeitgestaltung der nachwachsenden Generation, wobei besonders die ausserschulische Verwendung des Computers als Bildungsmittel an Bedeutung gewinnt (JIM 2010, Schmidt/Tippelt 2004, Treumann u. a. 2007). Auch in der Schule spielen die «Neuen Medien» verstärkt bei der Wissensvermittlung eine Rolle (Statistisches Bundesamt 2007, Krotz/Hasebrink 2002). Neben das pädagogisch strukturierte Schulbuch als traditionelles Leitmedium des Unterrichts (z. B. Gautschi 2007) sind andere Bildungs- und Freizeitmedien getreten, die die Deutungshoheit schulischer Bildungsvermittler, wie Bücher und Lehrkräfte, unterstützen aber auch kontrastieren können. Die Institution Schule als klassische Sozialisationsinstanz muss sich somit neuen Herausforderungen stellen, will sie ihrer Qualifikationsfunktion zur Ausbildung gesellschaftlich aktiv handlungsfähiger Individuen gerecht werden. Es ist davon auszugehen, dass sich der Status von Schulbuchwissen unter den Bedingungen derzeitiger Globalisierungsprozesse in zweierlei 
Hinsicht verändert hat: Zum einen wird die Kanonisierung von Wissen in Zeiten der Vervielfältigung von Zentren der Wissensproduktion zunehmend schwieriger. Die Geltungskraft und die normative Verbindlichkeit von nationalen Wissensordnungen nimmt in dem Masse ab, in dem sich Wissensproduzenten und -konsumenten der Tatsache bewusst werden, dass dieselben Tatbestände an anderen Orten auch anders konstruiert und wahrgenommen werden können. Zum anderen müssen schulische Bildungsmedien und das in ihnen repräsentierte Wissen im Zuge der globalen Medienrevolution verstärkt in Konkurrenz mit dem in anderen Medien transportierten Wissen treten. Das gilt insbesondere für das Internet, das im Zeitalter der Globalisierung zum Leitmedium geworden ist (Wagner 2002). In Folge dieser beiden Prozesse sind die Anforderungen an die Reflexionsfähigkeit, wie auch an die Medienkompetenz insgesamt (Baacke 1996), desjenigen, der mit dem in diesem Sinne kontingent gewordenen Wissen umgehen muss, erheblich gestiegen.

Unter diesen Bedingungen der multioptionalen, globalisierten Informationsgesellschaft, bei der alle Lebensbereiche zunehmend von Informations- und Kommunikationstechnologien durchdrungen werden (Castells 1996), ist kein Aufwachsen in pädagogisch geschützten Räumen mehr möglich (Baacke/Sander/Vollbrecht 1990). Daraus wird deutlich, dass Heranwachsende mit Kompetenzen zur Selbstbildung im Sinne des «Sich-Bildens der Persönlichkeit» (von Hentig 1996) ausgestattet sein müssen, wenn sie in der Informationsgesellschaft bestehen wollen. Das schliesst Medienkompetenz und Medienbildung als reflexive Auseinandersetzung mit Medien ein und bedeutet immer auch mediale (Selbst-) Sozialisation (Vollbrecht 2003). Dies führt wiederum dazu, dass sich die Aneignung von Weltwissen sowie Deutungs- und Handlungsmustern von direkten Bezugspersonen wie Eltern oder Lehrern zunehmend unabhängiger entwickelt, während der Einfluss medialer Orientierungsmuster und Vorbilder steigt (Fromme u. a. 1999, Tully 2004). Die Weitergabe von Kompetenzen erfolgt vor allem im Medienbereich zunehmend durch die Medien selbst wie auch durch die Peergruppe (Treumann u.a. 2007). Abseits unterhaltungsorientierter Mediennutzung gewinnt zudem die bildungsorientierte Rezeption medialer Angebote in Form informellen Lernens (Overwien 2005) entscheidende Bedeutung bei der Wahrnehmung von Lebenschancen und beruflichen Perspektiven. Dabei werden ausserschulische Bildungsaktivitäten umso wichtiger, je mehr schulische Abschlüsse an relativem Wert verlieren und Bildungsrenditen sinken (Stecher 2005).

Es kann also keineswegs angenommen werden, dass Schulbücher und Lehrkräfte die alleinigen Vermittler schulrelevanter Kompetenzen darstellen. Vielmehr wird es für Schüler/innen zunehmend notwendig, erstens Kompetenzen zur Selbstbildung 
durch Technik und Medien zu entwickeln (Tully 2004), die zu einer Lernkompetenz ${ }^{1}$ als grundlegender Qualifikation (Schmidt/Tippelt 2004) beitragen und zweitens konkrete schulrelevante Themen auch ausserhalb des Unterrichts mit Hilfe von (technischen) Bildungsmedien zu bearbeiten. Solche Prozesse der Selbstsozialisation und Selbstbildung stützen laut Tully (2004) auch den formalen Kompetenzerwerb Heranwachsender. Dieser oft behauptete Zusammenhang ist allerdings empirisch noch zu wenig untermauert worden. Dabei ist es zweckmässig, die benötigte fachliche Bindung von Kompetenzen über Themen herzustellen, die sowohl kanonisiert in Schulbüchern als auch auf vielfältige Weise im Internet behandelt werden und darüber hinaus Anknüpfungspunkte zu alltäglichen jugendlichen Lebenskontexten aufweisen. Zeitgeschichtliche Themen scheinen dafür besonders geeignet, denn sie können einerseits jugendliche Lebenswelten berühren und sind andererseits bereits Gegenstand von Schulbüchern (z. B. im Fach Geschichte). Die skizzierten Entwicklungen zeigen, dass informelles Lernen mit Medien weiter an Bedeutung gewonnen hat und dass Auswirkungen auf den formalen, schulischen Kompetenzerwerb anzunehmen sind. Eine Frage für zukünftige Forschungsvorhaben könnte daher lauten, inwieweit informelle Lernprozesse und hier insbesondere die Selbstbildung anhand unterrichtsrelevanter Themen mit Computer/ Internet und Schulbüchern, dazu geeignet sind, schulische Bildungsvermittler sinnvoll zu ergänzen und zu einem Kompetenzzuwachs bei Jugendlichen in einer schulischen Lernsituation beizutragen. Dabei ist aufgrund bisheriger Studien zu erwarten, dass sich die informelle Medienrezeption mit Schulbüchern und Computer/Internet wie auch die Lern- und thematischen Kompetenzen der Jugendlichen erstens nicht einheitlich darstellen (z. B. Prenzel u.a. 2008) und zweitens eingebettet sind in personale und situationale Rahmenbedingungen ihrer individuellen Lebenswelten (z. B. Treumann u. a. 2007). Dies legt es nahe, Unterschiede in den genannten Bereichen nicht nur aufzuzeigen, sondern vielmehr die Dimensionalität dieser komplexen Merkmale zu untersuchen und personenübergreifende Muster zu identifizieren, um diese in einer Typologie der schulrelevanten Medienrezeption zu integrieren. Eine daran anschliessende experimentelle Überprüfung des Kompetenzerwerbs Jugendlicher in Abhängigkeit von Rezeptionstyp und Bildungsmedium anhand eines schulrelevanten Themas würde deutlich machen, welcher Typ von Lernenden von welcher Lernumgebung am ehesten profitiert. Dies wiederum sollte auch zu einer Verbesserung der Gestaltung von Lernarrangements durch die Passung an Eingangsvoraussetzungen von Schüler/innen im Unterricht beitragen. Ziel dieses Beitrages ist es, erstens die aktuelle Medienrezeptionsforschung vor allem im Bereich bildungsorientierter Mediennutzung und den Forschungsstand zum Umgang mit Schulbüchern im Fach Geschichte zusammenfassend darzustel-

1 Lernkompetenz umfasst nach Tippelt u.a. (2003) Fähigkeiten zum selbstregulativen Lernen, zum kooperativen Lernen mit anderen und zum Umgang mit (Neuen) Medien. 
len, zu diskutieren und aus den jeweiligen Desideraten weitere Forschungsnotwendigkeiten zum Lernen mit Computer/Internet und Schulbüchern in Freizeit und Schule abzuleiten. Zweitens soll ein medienpädagogischer Forschungsansatz vorgestellt werden, bei dem versucht wird, beide Untersuchungsfelder so zu verbinden, dass die informelle schulrelevante Medienrezeption einschliesslich ihres Bedingungsgefüges sowie ihre Effekte auf den formalen Kompetenzerwerb mit diesen Medien aus Schülersicht untersucht werden können. Um die geschilderte Mehrdimensionalität des Gegenstands auch in einem Untersuchungsdesign abbilden zu können, wurde ein theoretischer Rahmen in Form eines Prozessmodells entwickelt, das unterschiedliche theoretische Ansätze triangulativ miteinander verknüpft und in ihrer spezifischen Bedeutung für ein mögliches empirisches Vorgehen darstellt.

\section{Empirische Medienrezeptions- und Schulbuchforschung und Schlussfolgerungen für ein triangulatives Forschungskonzept}

\section{Medienrezeptionsforschung}

In zahlreichen Studien zur Medienrezeption wurde gezeigt, dass Computer und Internet zum Alltag Jugendlicher gehören, zu vielfältigen Unterhaltungs-, Kommunikations- und Informationszwecken genutzt werden (z. B. JIM 2009, JIM 2010, Oehmichen/Schröter 2008, Shell 2006) und dass dabei Sozialisationsbedingungen der Nutzer eine beeinflussende Rolle spielen (Treumann u. a. 2007, Senkbeil/Wittwer 2008). Zwei der wichtigsten informationsorientierten Tätigkeiten am Computer und im Internet sind demnach das Arbeiten bzw. das Recherchieren von Informationen für die Schule. Allerdings ist über abstrakte Aktivitätsangaben hinaus vielfach offen geblieben, welche schulrelevanten Themen konkret in welcher Art und Weise bearbeitet werden. Qualität und Quantität der Mediennutzung Heranwachsender werden von personalen wie auch situationalen Faktoren beeinflusst. Neben demographischen Merkmalen wie Alter und Geschlecht haben sich das formale Bildungsniveau sowie das soziale und kulturelle Milieu der Familie als Einflussfaktoren auf die Mediennutzung erwiesen (Stecher 2005, Senkbeil/Wittwer 2008, Treumann u. a. 2007, Pietrass/Schmidt/Tippelt 2005). Die PISA-Studien haben darüber hinaus gezeigt, dass soziale Herkunft und Kompetenz in der Schule stark miteinander gekoppelt sind und soziale Ungleichheiten generationsübergreifend reproduziert werden (Baumert/Schümer 2001, Prenzel u. a. 2007, Senkbeil/Wittwer 2008). Daraus folgend stellt sich für weitere Forschungen die Frage, inwiefern das familiäre Milieu Jugendlicher auch eine erklärende Variable bzw. Bedingung für die Nutzung von schulrelevanten Medienangeboten in der Freizeit darstellt und ob ungünstige Ausgangslagen in Form sozialer Disparitäten durch selbstgesteu- 
erte Bildungsaktivitäten ausgeglichen werden können oder sich vorhandene Klüfte eventuell sogar vergrössern.

Treumann u. a. (2007) haben die Mediennutzung Jugendlicher thematisch breit anhand der Dimensionen des Bielefelder Medienkompetenzmodells (Baake 1996) analysiert, clusteranalytisch sieben Typen jugendlichen Medienhandelns erstellt und diese in den sozialen Kontext ihrer Lebenswelten eingeordnet. Die grössten Anteile stellen die Bildungsorientierten (20,4\%, meist Gymnasiastinnen) und die vorwiegend aus Arbeiterfamilien stammenden Positionslosen (20,3\%). Während sich erstere durch eine überdurchschnittliche Printmediennutzung auszeichnen und die Neuen Medien Computer/Internet zur Informationsrecherche und zum selbstgesteuerten Lernen verwenden, verhält es sich bei den Positionslosen umgekehrt: Printmedien nutzen sie kaum, Computer und Internet meist spielerisch. Ein weiteres Kontrastpaar bilden die Allrounder (12\%; überwiegend höher gebildet) und die Deprivierten (7,8\%; eher niedrig gebildet); erstere nutzen traditionelle und Neue Medien kompetent und zugleich überdurchschnittlich häufig, letztere dagegen überwiegend für rezeptiven Fernsehkonsum. Diese Klassifizierung nach differenzierenden Profilen des Medienhandelns stellt eine geeignete methodische Vorgehensweise dar, um interindividuelle Merkmale zu systematisieren. Der inhaltliche Schwerpunkt für die Untersuchung bildungsorientierter Medienrezeption müsste jedoch ein anderer sein: Es sollte eine Typologie zur schulrelevanten Medienrezeption erstellt werden, die neben überfachlichen und fachbezogenen Bildungsaktivitäten auch konkrete Themen des Unterrichts einbezieht. Somit würde jenseits unterhaltungsorientierter Mediennutzung das Lernen mit Schulbüchern und Computer/Internet in einem festgelegten Themenfeld, über Aktivitätsabfragen hinaus untersucht und in Zusammenhang mit personalen und sozialen Einflussfaktoren gestellt.

Die Art der Computernutzung in Freizeit und Schule scheint entscheidend für positive Effekte auf den fachlichen Kompetenzerwerb zu sein. Im Rahmen der PISA'06-Studie stellten Senkbeil und Wittwer (2007) fest, dass eine programmbezogene Nutzung des Computers (z. B. Lernprogramme) einen signifikant förderlichen Einfluss auf mathematische Kompetenzen von Schüler/innen aufweist. Leider ist Deutschland das OECD-Land mit dem seltensten Einsatz des Computers als Lernwerkzeug in der Schule. Dies verdeutlicht, neben der Forderung nach einer entsprechenden Anwendung im Unterricht, besonders die Notwendigkeit selbstgesteuerten Lernens in der Freizeit.

Weiter zeigten Senkbeil und Wittwer (2008) an Teilnehmern von PISA'06, dass die Art der Mediennutzung durch sozialisatorische Bedingungen beeinflusst wird und dass unterstützende und kommunikative Familienverhältnisse zu einer «anspruchsvolleren» Mediennutzung beitragen können. Diese wirke sich, im Gegensatz zu 
unterhaltungsbezogenen Medienaktivitäten, positiv auf schulische Kompetenzen in den Bereichen Naturwissenschaften und Lesen aus.

Detailliertere Analysen zur Nutzung von Computer und Internet als Bildungsmedien ${ }^{2}$, die sich nicht auf eine Abfrage von relativ abstrakten Nutzungsaktivitäten beschränken und neben der sozialen Herkunft der Rezipienten deren thematisches Interesse, den Austausch mit peers sowie psychologische Merkmale und kognitive Prozesse bzw. Aktivitäten während der Rezeption mitberücksichtigen und darüber hinaus untersuchen, wie sich die informelle Medienrezeption auf den formalen Kompetenzerwerb mit schulischen Bildungsmedien in einer spezifischen Domäne auswirken kann, fehlen bislang.

\section{Schulbuchforschung im Fach Geschichte}

Empirische Schulbuchforschung ist nach Einschätzung von Wiater (2003) in den letzten Jahren wieder verstärkt betrieben worden, hauptsächlich als Reaktion auf den wachsenden Konkurrenzdruck durch die Neuen Medien. Schulbuchforschung könne so als Teil der Medienforschung gesehen werden. Besonders die noch seltenen Rezeptionsanalysen zur Verwendung des Lehrbuchs in und ausserhalb der Schule, die überdies dessen Kontexte einbeziehen, sind für weitere Forschungsarbeiten zum Lernen mit Schulbüchern von Interesse.

Hier liegt eine Reihe von Studien zum Umgang mit Geschichtsschulbüchern überwiegend in der Schule vor. Besonders die hauptsächlich quantitativ befragend angelegten Studien von Bodo von Borries $(1992,1995,1999,2005)$ sind hier zu nennen. Aus ihnen, wie auch z. T. aus Arbeiten von Gautschi u. a. (2007), geht hervor, dass sie nach wie vor das Leitmedium des Unterrichts darstellen und dort vornehmlich lehrerzentriert eingesetzt werden. Ausserhalb des Unterrichts z. B. zur Vor- und Nachbereitung oder für Prüfungen spielen sie jedoch für Schüler/innen kaum eine bedeutsame Rolle. Schulbücher bleiben in ihrer wahrgenommenen Qualität weit hinter den Idealvorstellungen von Schülern und Lehrern vor allem hinsichtlich eingeforderter geschichtsdidaktisch relevanter Merkmale wie Multiperspektivität (Bergmann 2000) und Kontroversität (Grammes 2005) zurück und werden nur sehr verhalten und eher als «notwendiges Übel» (von Borries 2006) beurteilt. Darüber hinaus scheint es nach von Borries eine starke Überforderung durch Lehrbuchinhalte zu geben, denn sie werden von einem grossen Teil der Schüler/innen bei Wissensabfragen und in noch grösserem Masse bei Weiterverarbeitung und Beurteilung nicht richtig verstanden ${ }^{3}$. Als Ergebnis einer kombiniert

2 Kübler (1997) bezeichnet in einem engeren Sinne solche Medien als Bildungsmedien, die entweder in einem pädagogischen Zusammenhang eingesetzt oder mit dessen Hilfe Bildungsprozesse initiiert werden. Dabei kann es sich um formale (z. B. Schule), nonformale (z. B. ausserschulische Bildung) oder informelle Bildungskontexte (nondirektionales, selbstgesteuertes Lernen) handeln.

3 Bei der Beantwortung von Fragen zum Text verblieben im Rahmen eines Experiments Sechst- und Siebtklässler bei der Ratewahrscheinlichkeit und auch Schüler/innen der elften und zwölften Klassen 
befragend und experimentell vorgehenden Studie zu Ergebnissen des Geschichtsunterrichts kommt von Borries (2005) mittels Clusteranalysen zur Bildung von fünf Lerner- und Leistungstypen. Als Kriterien wurden u. a. historische Wissensaspekte sowie Wertungen und Deutungen zu geschichtlichen Themen (z. B. Bonifatius) herangezogen. Die ermittelten Typen reichen von geschichtsvertrauten Gutinformierten, über historisierende Kritische, skeptisch-geschichtsreservierte Unauffällige, naive Ja-Sager bis hin zu geschichtsfremden Uninformierten. Leider scheint das Thema «Bonifatius» recht weit von jugendlichen Lebenswelten entfernt, was die unbefriedigenden Schülerleistungen mit erklären könnte. Ausserdem wurden in den Studien, z. B. von v. Borries, noch zu wenig Zusammenhänge zu personalen und sozialen Lernermerkmalen hergestellt, die Hinweise auf Ursachen für die unbefriedigende Schulbuchverwendung und -verständlichkeit geben könnten. Daher wird hier die Notwendigkeit detaillierterer empirischer Untersuchungen zum Lernen mit Schulbüchern sowie den begleitenden psychologischen und sozialen Einflussgrössen deutlich.

Eine explorative Untersuchung zur Erfassung der historischen Perspektivenübernahme als Teilkompetenz historischen Denkens führten Hartmann, Sauer und Hasselhorn (2009) durch. Diese wurde durch die Bewertung von gegebenen Aussagen zu einem lehrplanbezogenen fiktiven Szenario erhoben. Dabei zeigten sich für Gymnasiasten der zehnten Klasse signifikante, positive Zusammenhänge zwischen der Kompetenz der historischen Perspektivenübernahme und fachspezifischen Merkmalen des Geschichtsunterrichts (u. a. fachliches Interesse, fachbezogenes Selbstkonzept, Geschichtsnote). Die Autoren resümieren, dass sich die historische Perspektivenübernahme in das Beziehungsgefüge fachspezifischer Leistungs- und Motivationsindikatoren einreiht, verweisen allerdings mit dem Hinweis auf Grenzen ihrer Studie (u. a. Validitätsprobleme, Aufgabenformate) auf die Notwendigkeit weiterer Forschung zur Erfassung von (Teil-) Kompetenzen historischen Denkens. Sauer spricht von Desideraten, wenn es erstens um die empirische Überprüfung vorhandener Modelle und zweitens um die Frage nach schulischen Lernarrangements geht, die zur Kompetenzförderung geeignet sind (Sauer 2006).

Insgesamt lässt sich feststellen, dass empirische Schulbuchforschung noch nicht die Bedeutung besitzt, die ihr aus schulischer Perspektive zukommen sollte (Fuchs/Kahlert/Sandfuchs 2010, Wiater 2003). Als Forschungsdesiderata identifiziert Wiater u. a. Rezeptions- und Wirkungsanalysen zur Verwendung des Schulbuchs und Studien, die das Lehrbuch im Kontext anderer Medien untersuchen. Auch Heinze (2010) weist auf die Notwendigkeit hin, wichtige Kontextbereiche wie den pädagogischen Verwertungszusammenhang des Schulbuchs in zukünftige Untersuchungen einzubeziehen. Demnach führe eine methodisch auf Inhaltsana-

konnten nur etwas mehr als die Hälfte der Aufgaben richtig lösen. Zu selbständiger Sinnbildung gelangten nur wenige Schüler (von Borries 2006). 
lysen beschränkte Schulbuchforschung zu einer verkürzten Perspektive und überschätze die Wirkung dieses Mediums. Dies betrifft auch den Aneignungsprozess des Schulbuchwissens, bei dem häufig eine lineare Wirkung des Schulbuchs vom Inhalt auf die Rezipienten/innen unterstellt wird. Von Borries (1999) hält weitere Untersuchungen zu konkreten historischen Themen für notwendig und schlägt vor, Unterschiede zwischen Lernenden zur Bildung einer Typologie zu nutzen. Über Nutzung und Wahrnehmung des Schulbuchs im Unterricht hinaus gibt es bisher kaum Studien, die das Lehrbuch ausserhalb der Schule und vor allem im Kontext anderer schulrelevanter Medien, wie z. B. Computer/Internet, sowie in seiner Bedeutung für den jugendlichen Kompetenzerwerb in einer bestimmten Domäne und in Abhängigkeit von unterschiedlichen Rezeptionstypen untersucht haben.

\section{Thematische und methodische Schlussfolgerungen für ein Forschungskonzept}

Die bisherigen Arbeiten im Bereich der Medienrezeptionsforschung und die Studien zu historischem Lernen mit Schulbüchern lassen Forschungsdesiderate erkennen, wenn es um die Untersuchung ausserschulischer Selbstbildungsaktivitäten mit Bildungsmedien und deren Einflüsse auf den schulischen Kompetenzerwerb mit Medien geht. Sie bieten jedoch wichtige thematische und methodische Anknüpfungspunkte für ein triangulatives Forschungskonzept, mit dem die informelle schulrelevante Medienrezeption einschliesslich ihres Bedingungsgefüges sowie ihre Effekte auf den formalen Kompetenzerwerb umfassend untersucht werden kann.

Dies gilt erstens für die gegenüber einer reinen Abbildung von Unterschieden anschaulichere und ökonomischere Typenbildung bei der Medienrezeption und dem Einbezug von Merkmalen jugendlicher Lebenswelten. An das methodische Vorgehen bei der Typenbildung von Treumann, Arens und Ganguin (2010) anknüpfend, wird in diesem Konzept eine thematische Erweiterung vorgenommen: Es sollen ausserschulische mediale Bildungsaktivitäten und informell erworbene Kompetenzen aus dem Themenspektrum des Geschichtsunterrichts standardisiert erhoben und mit bedeutsamen Einflussfaktoren aus der Lebenswelt Jugendlicher in Beziehung gesetzt werden. Denn es wurde bisher kaum erforscht, zu welchen Bildungsaktivitäten Heranwachsende Schulbücher und Computer/Internet in dieser Lerndomäne nutzen, welche schulrelevanten Medienangebote sie auf welche Art und Weise rezipieren, welche Funktionen sie diesen Medien zuschreiben, welche Einstellungen ihnen gegenüber bestehen, welche Kompetenzen bei Themen des Geschichtsunterrichts feststellbar sind und welche Rolle die soziale Herkunft dabei spielt. Hierbei sollten zur breiteren Analyse des informellen Lernens über verschiedene schulrelevante Medien hinweg auch Nutzungsaspekte des in jugendlichen Lebenswelten bedeutsamen Fernsehens sowie von Computerspielen besonders mit historischen Themen einbezogen werden (z. B. Stellenwert und Funktionen 
einzelner Medien im Medienmenü). Um diese sonst isoliert stehenden Variablen personenübergreifend systematisieren und mittels strukturentdeckender und klassifizierender Verfahren zu Profilen zusammenführen zu können, erscheint eine empirisch begründete Typenbildung der schulrelevanten informellen Medienrezeption als geeignete Vorgehensweise. Ein Typ repräsentiert demnach rezeptionsrelevante Strukturmerkmale bzw. Merkmalskombinationen, die ihn von anderen Typen unterscheiden. Diese Typologie stellt dann wiederum als eine unabhängige Variable die Vorrausetzung für eine experimentelle Überprüfung der Kompetenzen von Schüler/innen dar. Damit kann das Forschungsvorhaben zu einer umfassenderen und systematisch strukturierenden Analyse der ausserschulischen Rezeption von Computer und Internet als Bildungsmedien, auch im Vergleich zu anderen Medien jugendlicher Lebenswelten, beitragen.

Zweitens werden die Arbeiten von Borries' insofern aufgegriffen, als dass Sachkompetenzen sowie Deutungs- und Reflexionsmuster Jugendlicher gegenüber Themen des Geschichtsunterrichts in einer schulischen Lernsituation experimentell erfasst werden sollen. Allerdings bezieht dieses Konzept neben dem Lernen mit dem Schulbuch auch das Lernen mit Computer/Internet ein, es wird also eine mediale Erweiterung vorgenommen. Die experimentelle Untersuchung des Kompetenzerwerbs mit diesen Medien sollte in erster Linie unter einer pädagogisch-didaktischen Perspektive erfolgen. Drei Alternativen scheinen hier möglich: Erstens könnte das pädagogisch strukturierte und didaktisch aufbereitete Schulbuch einer freien, uneingeschränkten Nutzung des Internets gegenüber gestellt werden (mit Log-File-Analysen zur Erfassung tatsächlicher Internetaktivitäten). Dies entspräche eher der Verwendung in der schulischen Praxis, könnte aber eine Konfundierung der Variablen Medien und Abgeschlossenheit des Suchraums bedeuten, denn die Schulbuchgruppe wäre auf das Buch festgelegt, die Internetgruppe jedoch frei. Zweitens könnten dem Schulbuch speziell aufbereitete themenspezifische Internetseiten oder Ausschnitte aus einem Computerlernprogramm gegenübergestellt werden. Dies würde aber nur eine mögliche Form der Verwendung dieser Medien, besonders des Internets, bedeuten und die alltagspraktische Nutzung nicht mehr adäquat abbilden. Drittens könnte die Schulbuchgruppe weitere, frei wählbare Printmedien (z. B. aus der Schulbibliothek oder zusätzliche Arbeitsblätter) verwenden ${ }^{4}$, die Internetnutzung wäre hier ebenfalls frei. Dies würde bei vergleichbaren potentiellen Freiheitsgraden beider Gruppen in der Mediennutzung erstens einen Schwerpunkt auf die Analyse der faktischen Ausnutzung dieser Optionen bzw. Potentiale legen. Zweitens könnten die möglichen medial bedingten Unterschiede in der Zugangsweise sowie der methodisch-didaktischen und inhaltlichen Aufbe-

4 Auch hier sollte die tatsächliche Verwendung der Materialien durch die Schüler erfasst werden. 
reitung der Themen und deren Bedeutungen für den individuellen Kompetenzerwerb untersucht werden.

Weiterhin sollte die Prüfung des Kompetenzerwerbs in Abhängigkeit von den zuvor gebildeten Rezeptionstypen erfolgen. Damit kann untersucht werden, welche Rezeptionstypen mit unterschiedlichen Eingangsvoraussetzungen diese Angebote auch tatsächlich nutzen und welchen Lernerfolg verschiedene Typen mit Schulbuch bzw. Computer/Internet erreichen. Zum Beispiel wäre von Interesse, ob die dem Schulbuch als unzureichend vorhanden unterstellte Multiperspektivität besser durch das Lernen mit dem Internet geleistet werden kann und welche Rezeptionstypen damit eher zu multiperspektivischen Kompetenzen gelangen können und welche weniger. Somit könnten die bislang nicht untersuchten Effekte informellen Lernens mit diesen Medien unter Berücksichtigung situativer und personenbezogener Eingangsvoraussetzungen auf den Kompetenzerwerb mit ihnen in einem schulischen Setting analysiert werden.

Insgesamt wird mit diesem Forschungskonzept versucht, bisherige Ansätze aus standardisierter Befragung mit anschliessender Typenbildung und experimenteller Untersuchung in der Schule symbiotisch miteinander zu verknüpfen: Es werden Zusammenhänge zwischen schulrelevanter Medienrezeption in der Freizeit und formalem Kompetenzerwerb mit Bildungsmedien in der Schule anhand thematischer Beispiele hergestellt, die sowohl im Geschichtsbuch behandelt werden als auch ausserschulische jugendliche Lebenskontexte berühren. Dabei werden gezielt das traditionelle Leitmedium des Unterrichts, das Schulbuch (z. B. Gautschi 2007, Rüsen 2008) und das Leitmedium der Globalisierung, der Computer mit einer seiner wichtigsten Anwendungen, dem Internet (Wagner 2002), in ihrer je spezifischen Bedeutung für den jugendlichen Kompetenzerwerb in den Mittelpunkt gestellt. Indem Teilkompetenzen historischen Denkens anhand eines thematischen Beispiels erfasst werden, würde das Vorhaben auch einen Beitrag zur geforderten empirischen Überprüfung historischer Kompetenzmodelle leisten. Darüber hinaus sollte es mit dieser Untersuchungsanlage möglich sein, Potentiale und Chancen aber auch Grenzen des selbstgesteuerten wie auch institutionalisierten Umgangs mit den ausgewählten Medien und deren Inhalten unter Berücksichtigung jugendlicher Rezeptionstypen zu analysieren. Auf Basis der Ergebnisse sollten in Verbindung zur praktischen Bildungsarbeit Handlungsempfehlungen erstens zur Ausbildung bzw. Stärkung von Selbstbildungsfähigkeiten und zu einem kompetenten Umgang mit medial vermittelten Bildungsangeboten in der Freizeit abgeleitet werden. Zweitens könnten Einsatzmöglichkeiten «neuer» Bildungsmedien wie Computer und Internet im Unterrichtsalltag in Bezug auf unterschiedliche Lerntypen und Lernprozessmerkmale dargestellt werden. Damit sollte ebenfalls deutlich gemacht werden, wie durch ausserschulische Bildungsaktivitäten auch der schulische Kompetenzerwerb zumindest im gewählten Themenspektrum ge- 
fördert werden kann. Auf diese Weise werden Reflexions- und Orientierungshilfen für Jugendliche, Eltern und Lehrende aufgezeigt, die helfen könnten, Rezeptionsklüften bei der schulrelevanten Mediennutzung und dem Kompetenzerwerb mit Bildungsmedien entgegenzuwirken.

In methodischer Hinsicht ist «objektiveren Messverfahren» gegenüber subjektiven Selbsteinschätzungen von Schüler/innen der Vorzug zu geben. Da der Fokus auf der Erfassung von Kompetenzen beim selbständigen Umgang mit Medieninhalten liegt, erscheint der Einsatz diagnostischer Tests in der Schule als eine geeignete Vorgehensweise. Als alternative oder ergänzende Methode wären auch Unterrichtsbeobachtungen denkbar, die allerdings eher für die Erfassung von Interaktionsprozessen im Unterrichtsgeschehen angebracht scheinen.

\section{Ziele und Fragestellungen eines Forschungskonzepts}

Aus den beschriebenen Desiderata und den daraus gezogenen Schlussfolgerungen ergeben sich Ziele und konkrete Fragestellungen, denen auf Grundlage des hier zu erörternden Konzepts in möglichen empirischen Untersuchungen nachgegangen werden kann: Ziel müsste es sein, die schulrelevante Medienrezeption in selbstgesteuerten (informellen) Lernprozessen und deren Auswirkungen auf den Kompetenzerwerb in einer schulischen (formalen) Lernsituation aus Schülersicht zu untersuchen und mit individuellen Dispositionen der Rezipienten sowie Kontextmerkmalen ihrer Lebenswelten in Beziehung zu setzen.

Zentrale Fragestellungen eines mehrperspektivischen Untersuchungsvorhabens können sein:

1. Zu welchen Bildungsaktivitäten nutzen Jugendliche Schulbücher und den Computer bzw. das Internet ausserhalb der Schule? Welche (geschichts)- unterrichtsrelevanten Medienangebote werden in welcher Art und Weise rezipiert? Welche Funktionen werden Schulbüchern und dem Computer/Internet zugeschrieben und welche Einstellungen bestehen ihnen gegenüber beim informellen Lernen? Welche historischen Sach- und Reflexionskompetenzen sind bei konkreten Themen des Geschichtsunterrichts feststellbar?

2. Welche Rezeptionstypen lassen sich dabei sinnvoll unterscheiden?

3. Welche Auswirkungen hat die unter Punkt 1 erfasste informelle Medienrezeption in Abhängigkeit vom Rezeptionstyp auf den formalen Kompetenzerwerb mit Computer/Internet und Schulbüchern in diesem Lernbereich?

4. Mit welchen situationalen und personalen Rahmenbedingungen jugendlicher Lebenswelten stehen die unter Punkt 1 erfassten Rezeptionsprozesse in Zusammenhang? 
In einem ersten Untersuchungsschritt müsste es das Ziel sein, mittels standardisierter Befragungen einen Überblick über die schulrelevante Medienrezeption Jugendlicher ausserhalb der Schule zu erhalten und in den Kontext ihrer Lebenswelten einzuordnen. Dazu gehören sowohl fachübergreifende Bildungsaktivitäten als auch die Rezeption geschichtsunterrichtsbezogener Angebote sowie die Erfassung von Kompetenzen historischen Denkens anhand der ausgewählten Themen. Auf dieser Basis sollen Unterschiede hinsichtlich der erfassten Merkmale nicht nur angezeigt, sondern vielmehr die Binnenstruktur der schulrelevanten Medienrezeption aufgedeckt werden, indem Hauptquellen von Unterschieden im jugendlichen Medienhandeln identifiziert und interindividuelle Rezeptionsmuster herausgearbeitet werden. Die Aufdeckung dieser Strukturen ermöglicht es schliesslich, eine empirisch fundierte Klassifizierung bzw. Typenbildung der informellen schulrelevanten Medienrezeption zu erstellen, aus der auch Handlungsempfehlungen für die pädagogische Praxis abgeleitet werden können.

In einem zweiten Schritt ist experimentell zu prüfen, welche thematischen Kompetenzen von den Untersuchungsteilnehmer/innen beim Lernen mit Computer/ Internet bzw. Schulbuch erreicht werden und ob und wenn ja, in welcher Art und Weise, sich die festgestellten unterschiedlichen Rezeptionstypen in ihrem Kompetenzerwerb je nach verwendetem Bildungsmedium unterscheiden; inwiefern differentielle Wechselwirkungseffekte zwischen Rezeptionstypen und Bildungsmedien feststellbar sind und ob bestimmte Typen eher vom Lernen mit einem der beiden Medien profitieren oder umgekehrt in ihrem Lernprozess behindert werden. Dadurch kann die Analyse des Kompetenzerwerbs auf die zuvor gebildeten Medienrezeptionsmuster rückbezogen werden.

\section{Theoretische Grundlagen und deren Triangulation in einem Prozessmodell}

Im hier vorgestellten Konzept soll versucht werden, differenzierte Fragestellungen in verschiedenen aber aufeinander bezogenen Bereichen des Umgangs mit Medien zu beantworten. Um diesem Vorhaben theoretisch und methodisch entsprechen zu können, ist es sinnvoll, sowohl unterschiedliche Erhebungsmethoden als auch theoretische Ansätze triangulativ miteinander zu kombinieren. Bei der Triangulation (Treumann 2005, Flick 2008) handelt es sich um eine Forschungsstrategie, mit der u. a. durch die Kombination unterschiedlicher wissenschaftlicher Disziplinen, Theorien, Methoden und Datenquellen umfassendere und vertiefende Erkenntnisse über soziale Phänomene gewonnen werden sollen. Daher wurde ein theoretischer Rahmen entworfen, der der Mehrdimensionalität des Untersuchungsgegenstands angemessen erscheint. So können empirisch festgestellte Ergebnisse vor dem Hintergrund theoretischer Konzepte interpretiert werden. Die wesentlichen Ansätze, die hier zu berücksichtigen sind, betreffen die Bereiche Medienkompetenz, Lernen mit Medien und dessen Einflussfaktoren sowie historisches Lernen. 
Den theoretischen Mittelpunkt bilden die Bereiche Mediennutzung und -kritik des Bielefelder Medienkompetenzmodells (Baacke 1996). Mithilfe der Nutzungsdimension kann über Häufigkeitsangaben erfasst werden, zu welchen Bildungsaktivitäten Jugendliche Schulbücher und Computer/Internet ausserhalb des Unterrichts nutzen und welche schulrelevanten Medienangebote sie wie rezipieren. Ausserdem sollte über die kritische Dimension auf einer abstrakteren Ebene nach reflexiven Einschätzungen des eigenen Medienhandelns und eigener Positionen sowie nach analytischen Bewertungen von Medien und -inhalten gefragt werden. Die notwendige domänenspezifische Operationalisierung erfolgt in diesem Konzept erstens über Medienaktivitäten mit thematischem Bezug zum Geschichtsunterricht. Hier wird der Uses-and-Gratifications-Approach zur Beschreibung der interessen- und bedürfnisgeleiteten Mediennutzung und deren kognitiven, sozialen und emotionalen Funktionen herangezogen (Blumler/Katz 1974). Zweitens sollten spezifische Teilbereiche historischen Denkens mit Hilfe des Kompetenzmodells des Verbandes der Geschichtslehrer Deutschlands (Sauer 2006) anhand exemplarischer Themen erhoben werden. Folgende Themen bieten sich dafür an:

- 1989: Das Ende des Ost-West-Konflikts

und der Beginn der «multipolaren Welt»

Dieses Ereignis ist im Geschichtsunterricht obligatorisch und die Wahrnehmungen der Teilung sowie die Zugehörigkeit zu "Ost» oder «West», weitergegeben durch Elternhaus, Schule oder Medien sind als Bestandteil der eigenen jugendlichen Identität anzusehen (Ferchhoff 2007). Es ist daher zu vermuten, dass das Thema sozialisatorische Bedeutung für den individuellen Lebenskontext von Jugendlichen besitzt. Dies wiederum kann die Rezeption des Ereignisses und den Kompetenzerwerb in diesem Bereich beeinflussen.

- 11. September 2001: Die Anschläge in den USA und der «Kampf gegen den Terror»

Dieses Ereignis wird zwar in Geschichtsschulbüchern behandelt, spielt aber im Lehrplan eine geringere Rolle. Es enthält zahlreiche medienpädagogische Implikationen. Die Beschäftigung damit lässt Bedingungen und Folgen, allgemein die Herausforderungen und Probleme der «Einen Welt» sichtbar werden. Es können Einsichten in die Funktionsweisen einer globalisierten (Medien-) Gesellschaft gewonnen werden, in der Medien Teil des Ereignisses sind und «Medienrealität» und «Alltagsrealität» zu verschwimmen scheinen (Moser 2006, Meister u. a. 2008).

Sauers Modell bildet die theoretische Grundlage sowohl für die Erfassung informell erworbener Kompetenzen als auch für den formalen Kompetenzerwerb. Aufgrund seiner mittleren Konkretionsebene und seiner pragmatisch-funktionalen 
Ausrichtung erscheint es für die empirische Erforschung von (Teil-) Kompetenzen als auch für die Messung und Förderung der Kompetenzentwicklung bei Schülern geeignet. Dabei erfolgt eine Beschränkung auf «Grundeinsichten in die Struktur historischen Fragens und Erkennens» (Sauer 2006). Folgende Teilbereiche kommen für eine empirische Erfassung besonders in Frage:

- Sachkompetenz

- Themenbezogene Sachkompetenz

- Orientierung in der Geschichte

- Deutungs- und Reflexionskompetenz

- Konstruktcharakter von Geschichte erkennen

- Mit Perspektivität in der Geschichte umgehen

Da Bildungsprozesse Jugendlicher mit Medien untersucht werden, ist die konstruktivistische Lerntheorie mit ihrer Grundannahme einer individuellen und prozesshaften Konstruktion von Wissen besonders geeignet, diese zu beschreiben und zu erklären (z. B. Steiner 2006, Schmidt/Tippelt 2004). Das konstruktivistische Paradigma dient hier als theoretischer Rahmen, innerhalb dessen das schulrelevante Medienhandeln insgesamt untersucht wird. Unter dieser Perspektive liegt das Potential von Bildungsmedien in ihrer Nutzung als kognitives Werkzeug. Der Fokus liegt dabei auf der selbständigen Erarbeitung von Problemstellungen und dem Prüfen einer Vielzahl möglicher Lösungswege. Des Weiteren ist beim konstruktivistischen Paradigma die Situiertheit des Wissenserwerbs von zentraler Relevanz. Das bedeutet für dieses Vorhaben, das Bedingungsgefüge der Medienrezeption einzubeziehen, da sich das Lernen mit Medien nicht losgelöst von personalen und situationalen Merkmalen individueller Lebenswelten vollzieht (Schaumburg/lssing 2004). Aufgrund bisheriger Befunde ist davon auszugehen, dass Unterschiede in den situativen Rahmenbedingungen des Lebenskontexts Jugendlicher (Merkmale des Elternhauses, makrosoziale Merkmale) sowohl Medienrezeption als auch Kompetenzerwerb beeinflussen. Ihnen und ihrem Zusammenspiel mit personalen Faktoren (soziodemographische Merkmale; ökonomisches, kulturelles und soziales Kapital) kommt besonders in der konstruktivistischen Lerntheorie entscheidende Bedeutung für den Lernerfolg zu. Personenbezogene Ressourcen dienen der Erklärung von Unterschieden beim Umgang mit Medien und können hinsichtlich der Kapitalsorten mithilfe des Habitusansatzes (Bourdieu 1983) beschrieben und empirisch fassbar gemacht werden.

Weiterhin ist aus bisherigen Studien bekannt, dass kognitive und affektive Einstellungen von Schüler/innen gegenüber den zu untersuchenden Medien als solches wie auch gegenüber konkreten Themen sowohl die informelle Medienrezeption 
als auch den formalen Kompetenzerwerb beeinflussen (z. B. Schulte/Bögeholz/ Watermann 2008, Frey u. a. 2009). Daher sollten medienspezifische Selbstwirksamkeitserwartungen (Bandura 1997, Fuchs 2005) beim informellen Lernen mit Computer/Internet und Geschichtsschulbuch sowie beim formalen Kompetenzerwerb die wahrgenommene Schwierigkeit der Aufgabenstellung und die investierte kognitive Anstrengung (Salomon 1984, Mauch 2007) sowie lernrelevante Emotionen als «lernprozessbegleitende Merkmale» (Wild/Hofer/Pekrun 2006) erhoben werden. Insgesamt zeigen Untersuchungen, dass die schulrelevante Medienrezeption bei Jugendlichen von einem Bedingungsgefüge bestehend aus verschiedenen Faktoren beeinflusst wird und sich ihrerseits im Zusammenspiel mit diesen Merkmalen auf den Kompetenzerwerb von Schüler/innen auswirkt. Letzterer wiederum steht ebenfalls in Zusammenhang mit lernprozessbegleitenden Faktoren. Nur wenn diese miteinander verwobenen Aspekte mit berücksichtigt werden, ist es möglich, belastbare, weil in den persönlichen und situativen Lebenskontext Jugendlicher eingebettete, empirische Aussagen über Medienrezeption und deren Effekte auf den Kompetenzerwerb in einer bestimmten Lerndomäne treffen zu können. Zur Analyse dieser komplexen Phänomene werden in dem hier vorgeschlagenen Konzept unterschiedliche theoretische Modelle genutzt, die, für sich genommen, je einen inhaltlich bestimmten Ausschnitt jugendlichen Medienhandelns zu erklären beanspruchen. Hier werden sie jedoch miteinander in der geschilderten Art und Weise im Sinne einer gegenseitigen Ergänzung miteinander verknüpft. Ziel ist eine umfassendere, theoretisch fundierte Analyse der Bedingungen und Effekte der schulrelevanten Medienrezeption Jugendlicher. Die Strategie der Triangulation scheint geeignet, der Mehrdimensionalität des Forschungsgegenstandes auch methodisch im Untersuchungsdesign durch die Kombination von standardisierter Befragung (informelle Medienrezeption) und Feldexperiment (formaler Kompetenzerwerb) gerecht werden zu können. Die folgende Abbildung stellt den theoretischen Rahmen dieses Ansatzes in Form eines Prozessmodells dar: 


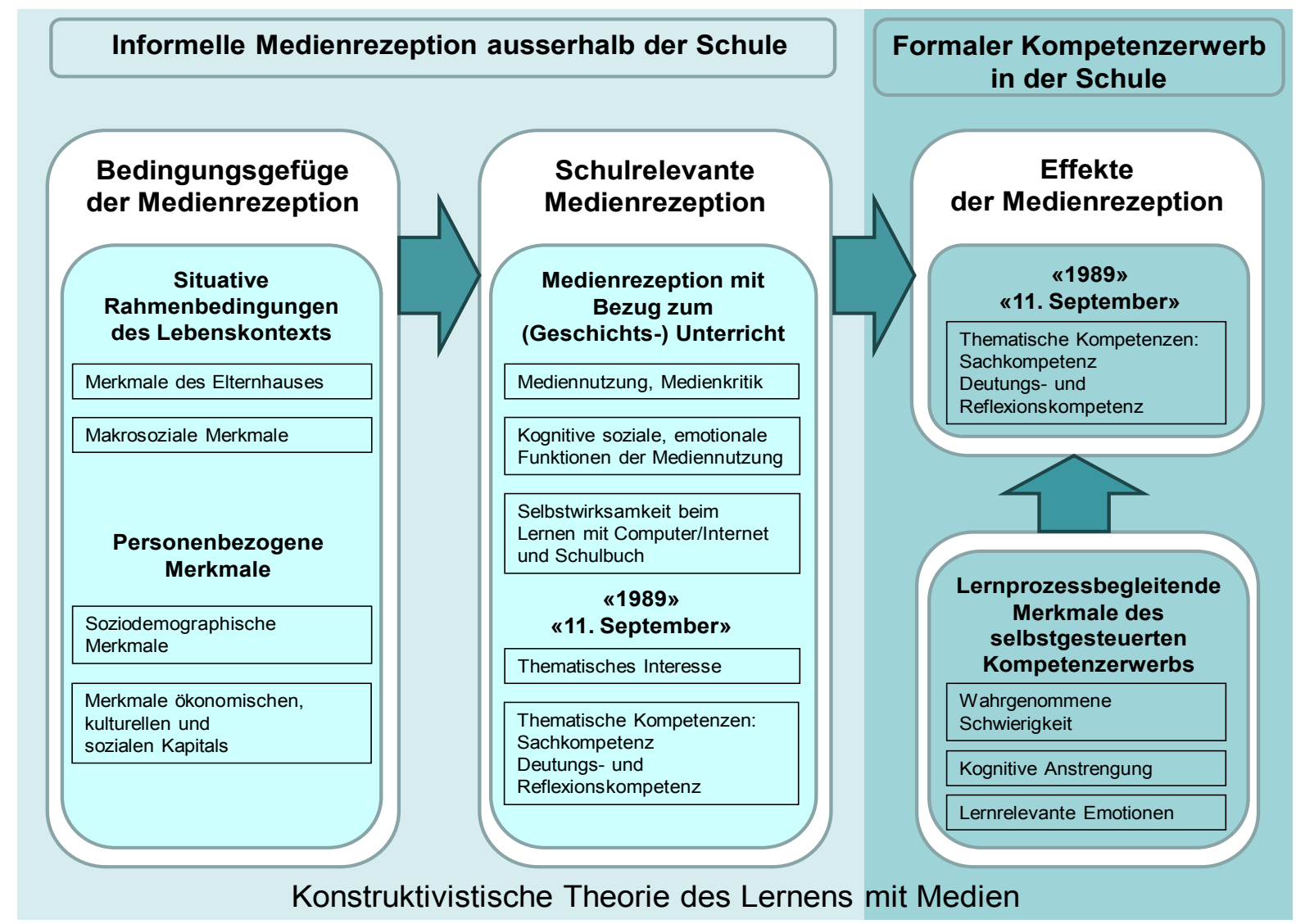

Abbildung 1: Theoretischer Rahmen in Form eines Prozessmodells

\section{Fazit}

Mit dem hier zur Diskussion gestellten Forschungskonzept sollte es möglich sein, Bedingungen und Effekte schulrelevanter Medienrezeption aus Schülersicht empirisch zu untersuchen. Dies könnte am Beispiel zweier zeitgeschichtlicher Themen aus dem Geschichtsunterricht: 1989: Das Ende des Ost-West-Konflikts und 11. September 2001: Die Anschläge in den USA erfolgen. Im Mittelpunkt stehen hierbei das Schulbuch als Leitmedium des Unterrichts sowie der Computer als Leitmedium der Globalisierung und somit Themen und Medien schulischer und ausserschulischer jugendlicher Lebenswelten. Mit diesem Ansatz wird der in der aktuellen Bildungsforschung relevanten Frage nachgegangen, inwieweit informelle Lernprozesse mit Computer/Internet und Schulbüchern den formalen Kompetenzerwerb mit diesen Medien beeinflussen können. Dazu können zwei Teilstudien durchgeführt werden: Ziel der ersten Teilstudie, einer quantitativen Fragebogenerhebung, sollte es sein, erstens einen breiten Überblick über die informelle Medienrezeption mit Bezug zum (Geschichts-) Unterricht zu erhalten und erwor- 
bene Sach- und Reflexionskompetenzen von Schüler/innen am Beispiel der zwei genannten Themen zu erfassen. Auf dieser Basis würde zweitens eine Typologie der informellen schulrelevanten Medienrezeption in diesem Themenfeld erstellt. In der zweiten Teilstudie sollten die gebildeten Rezeptionstypen als unabhängige Variable in eine experimentelle Studie eingehen. Darin würde der formale Kompetenzerwerb von Schüler/innen mit Schulbuchmaterialien und Internetangeboten in einer schulischen Lernsituation anhand beider oder eines der beiden Themen erhoben. Hauptziel wäre es, zu prüfen, ob differentielle Wechselwirkungseffekte zwischen Rezeptionstypen und Bildungsmedien beim Kompetenzerwerb feststellbar sind und ob bestimmte Typen aufgrund ihrer Eingangsvoraussetzungen eher vom Lernen mit dem Schulbuch oder Computer/Internet profitieren. Insgesamt sollte mit diesem Konzept eine umfassendere und tiefer gehende Analyse der schulrelevanten Medienrezeption einschliesslich ihres Bedingungsgefüges aus situativen und personalen Einflussfaktoren sowie ihrer Auswirkungen auf den Kompetenzerwerb Jugendlicher in einem spezifischen Lernbereich des Geschichtsunterrichts möglich werden.

\section{Literatur}

Bandura, Albert (1997). Self-Efficacy: The Exercise of Control. New York: Freeman.

Baacke, Dieter (1996). Medienkompetenz - Begrifflichkeit und sozialer Wandel. In: Rein, Antje von (Hrsg.), Medienkompetenz als Schlüsselbegriff. Bad Heilbrunn: Klinkhardt, S. 112-124.

Baacke, Dieter; Sander, Uwe; Vollbrecht, Ralf (1990). Lebenswelten sind Medienwelten. Opladen: Leske + Budrich.

Baumert, Jürgen; Schümer, Gundel (2001). Familiäre Lebensverhältnisse, Bildungsbeteiligung und Kompetenzerwerb. In: Baumert, Jürgen u. a. (Hrsg), PISA 2000: Basiskompetenzen von Schülerinnen und Schülern im internationalen Vergleich. Opladen: Leske + Budrich, S. 323-407.

Bergmann, Klaus (2000). Multiperspektivität. Geschichte selber denken, Schwalbach/Ts.: Wochenschau-Verlag.

Blumler, Jay G.; Katz, Elihu (Ed.) (1974). The Uses of Mass Communications: Current Perspectives on Gratifications Research. Beverly Hills: Sage Publications.

Borries, Bodo von (2006). Schulbuch-Gestaltung und Schulbuch-Benutzung im Fach Geschichte: Zwischen empirischen Befunden und normativen Überlegungen. In: Handro, Saskia; Schönemann, Bernd (Hrsg.), Geschichtsdidaktische Schulbuchforschung. Münster: LIT, S. 39-51.

Borries, Bodo von et al. (1992). Kindlich-jugendliche Geschichtsverarbeitung in West- und Ostdeutschland 1990: Ein empirischer Vergleich. Pfaffenweiler: Centaurus.

Borries, Bodo von et al. (1995). Das Geschichtsbewusstsein Jugendlicher: Erste repräsentative Untersuchungen über Vergangenheitsdeutungen, Gegenwartswahrnehmungen und Zukunftserwartungen in Ost- und Westdeutschland. Weinheim: Juventa.

Borries, Bodo von et al. (1999). Jugend und Geschichte: Ein europäischer Kulturvergleich aus deutscher Sicht. Opladen: Leske + Budrich. 
Borries, Bodo von et al. (2005). Schulbuchverständnis, Richtlinienbenutzung und Reflexionsprozesse im Geschichtsunterricht: Eine qualitativ-quantitative Schüler- und Lehrerbefragung im Deutschsprachigen Bildungswesen 2002. Neuried: ars una.

Bourdieu, Pierre (1983). Ökonomisches Kapital, kulturelles Kapital, soziales Kapital. In: Kreckel, Reinhard (Hrsg.), Soziale Ungleichheiten. Göttingen: Verlag Otto Schwarz \& Co., S. 183-198.

Castells, Manuel (1996). The Information Age: Economy, Society and Culture. Vol. 1: The Rise of the Network Society. Blackwell: Cambridge.

Ferchhoff, Wilfried (2007). Jugend und Jugendkulturen im 21. Jahrhundert. Wiesbaden: VS Verlag für Sozialwissenschaften.

Flick, Uwe (2008). Triangulation: Eine Einführung. Wiesbaden: VS Verlag für Sozialwissenschaften.

Frey, Andreas; Taskinen, Päivi; Schütte, Kerstin; Prenzel, Manfred; Artelt, Cordula; Baumert, Jürgen; Blum, Werner; Hammann, Marcus; Klieme, Eckhard; Pekrun, Reinhard (Hrsg.) (2009). Pisa '06: PISA 2006 Skalenhandbuch. Dokumentation der Erhebungsinstrumente. Münster: Waxmann.

Fuchs, Carina (2005). Selbstwirksam lernen im schulischen Kontext: Kennzeichen - Bedingungen - Umsetzungsbeispiele. Bad Heilbrunn: Klinkhardt.

Fuchs, Eckhardt; Kahlert, Jürgen; Sandfuchs, Uwe (Hrsg.) (2010). Schulbuch konkret. Kontexte, Produktion, Unterricht. Bad Heilbrunn: Klinkhardt.

Gautschi, Peter; Moser, Daniel; Reusser Kurt; Wiher, Pit (Hrsg.) (2007). Geschichtsunterricht heute: Eine empirische Analyse ausgewäh/ter Aspekte. Bern: h.e.p.

Grammes, Tilmann (2005). Kontroversität. In: Sander, Wolfgang (Hrsg.), Handbuch politische Bildung, Bonn: BpB, S. 126-145.

Hartmann, Ute; Sauer, Michael; Hasselhorn, Marcus (2009). Perspektivenübernahme als Kompetenz für den Geschichtsunterricht: Theoretische und empirische Zusammenhänge zwischen fachspezifischen und sozial-kognitiven Schülermerkmalen. In: Zeitschrift für Erziehungswissenschaft, 12.2, Wiesbaden: VS Verlag für Sozialwissenschaften, S. 321342.

Heinze, Carsten (2010). Historical Textbook Research: Textbooks in the Context of the «Grammar of Schooling». In: Journal of Educational Media, Memory, and Society, 2.2, Biggleswade: Berghahn Journals, 122-131.

JIM (2009). Medienpädagogischer Forschungsverbund Südwest (Hrsg.), Jugend, Information, (Multi-) Media: Basisuntersuchung zum Medienumgang 12- bis 19-Jähriger. Stuttgart: MPFS.

JIM (2010). Medienpädagogischer Forschungsverbund Südwest (Hrsg.), Jugend, Information, (Multi-) Media: Basisuntersuchung zum Medienumgang 12- bis 19-Jähriger. Stuttgart: MPFS.

Krotz, Friedrich; Hasebrink, Uwe (2002). Medienkompetenz von Kindern und Jugendlichen für die Informationsgesellschaft und ihre Bedingungen in Japan und Deutschland. Hamburg: Verlag Hans-Bredow-Institut.

Kübler, Hans-Dieter (1997). Bildungsmedien. In: Hüther, Jürgen; Schorb, Bernd; BrehmKlotz, Christiane (Hrsg.), Grundbegriffe Medienpädagogik. München: kopaed, S. 40-47.

Mauch, Martina (2007). Das Internet als Lernmedium: Wie Einstellungen zu Medien das Lernen beeinflussen. Saarbrücken: VDM Verlag Dr. Müller. 
Meister, Dorothee M.; Sander, Uwe; Treumann, Klaus Peter; Burkatzki, Eckhard; Hagedorn, Jörg (2008). Mediale Gewalt: Ihre Rezeption, Wahrnehmung und Bewertung durch Jugendliche. Wiesbaden: VS Verlag für Sozialwissenschaften.

Moser, Heinz (2006). Einführung in die Medienpädagogik: Aufwachsen im Medienzeitalter. Wiesbaden: VS Verlag für Sozialwissenschaften.

Oehmichen, Ekkehardt; Schröter, Christian (2008). Medienübergreifende Nutzungsmuster: Struktur- und Funktionsverschiebungen. In: Media Perspektiven, 8, Frankfurt/M., S. 394409.

Overwien, Bernd (2005). Stichwort: Informelles Lernen. In: Zeitschrift für Erziehungswissenschaft, 3, Wiesbaden: VS Verlag für Sozialwissenschaften, S. 339-355.

Pietrass, Manuela; Schmidt, Bernhard; Tippelt, Rudolf (2005). Informelles Lernen und Medienbildung: Zur Bedeutung sozio-kultureller Voraussetzungen. In: Zeitschrift für Erziehungswissenschaft, 03/2005, Wiesbaden: VS Verlag für Sozialwissenschaften, S. 412-426.

Prenzel, Manfred; Artelt, Cordula; Baumert, Jürgen; Blum, Werner; Hammann, Marcus; Klieme, Eckhard; Pekrun, Reinhard (Hrsg.) (2007). PISA 2006: Die Ergebnisse der dritten internationalen Vergleichsstudie. Münster: Waxmann.

Prenzel, Manfred; Artelt, Cordula; Baumert, Jürgen; Blum, Werner; Hammann, Marcus; Klieme, Eckhard; Pekrun, Reinhard (Hrsg.) (2008). PISA 2006 in Deutschland: Die Kompetenzen der Jugendlichen im dritten Ländervergleich. Münster: Waxmann.

Rüsen, Jörn (2008). Historisches Lernen: Grundlagen und Paradigmen. Schwalbach: Wochenschau.

Salomon, Gavriel (1984). Television is "easy» and print is "tough»: The differential investment of mental effort in learning as a function of perceptions and attributions. In: Journal of Educational Psychology, 76, pp. 647-658.

Sauer, Michael (2006). Kompetenzen für den Geschichtsunterricht - ein pragmatisches Modell als Basis für die Bildungsstandards des Verbandes der Geschichtslehrer. In: Informationen für den Geschichts- und Gemeinschaftskundelehrer, 72, Schwalbach/Ts.: Wochenschau-Verlag, S. 7-20.

Schaumburg, Heike; Issing, Ludwig J. (2004). Interaktives Lernen mit Multimedia. In: Mangold, Roland u.a. (Hrsg.), Lehrbuch der Medienpsychologie. Göttingen: Hogrefe, S. 717-742.

Schmidt, Bernhard; Tippelt, Rudolf (2004). Multimediale Lernangebote und ihre Eignung für Jugendliche. In: Tully, Claus J. (Hrsg.), Verändertes Lernen in modernen technisierten Welten: Organisierter und informeller Kompetenzerwerb Jugendlicher. Wiesbaden: VS Verlag für Sozialwissenschaften. S. 87-106.

Schulte, Klaudia; Bögeholz, Susanne; Watermann, Rainer (2008). Selbstwirksamkeitserwartungen und Pädagogisches Professionswissen im Verlauf des Lehramtsstudiums. In: Zeitschrift für Erziehungswissenschaft, 2, Wiesbaden: VS Verlag für Sozialwissenschaften, S. 268-287.

Senkbeil, Martin; Wittwer, Jörg (2007). Die Computervertrautheit von Jugendlichen und Wirkungen der Computernutzung auf den fachlichen Kompetenzerwerb. In: Prenzel, Manfred; Artelt, Cordula; Baumert, Jürgen; Blum, Werner; Hammann, Marcus; Klieme, Eckhard; Pekrun, Reinhard (Hrsg.), PISA 2006: Die Ergebnisse der dritten internationalen Vergleichsstudie. Münster: Waxmann, S. 277-307.

Senkbeil, Martin; Wittwer, Jörg (2008). Antezedenzien und Konsequenzen informellen Lernens am Beispiel der Mediennutzung von Jugendlichen. In: Prenzel, Manfred; Baumert, 
Jürgen (Hrsg), Vertiefende Analysen zu PISA 2006, Zeitschrift für Erziehungswissenschaft, Sonderheft 10, Wiesbaden: VS Verlag für Sozialwissenschaften, S. 107-128.

Statistisches Bundesamt (2007). Entwicklung der Informationsgesellschaft. IKT in Deutschland. Ausgabe 2007. Wiesbaden: Statistisches Bundesamt.

Stecher, Ludwig (2005). Informelles Lernen bei Kindern und Jugendlichen und die Reproduktion sozialer Ungleichheit. In: Zeitschrift für Erziehungswissenschaft, 3, Wiesbaden: VS Verlag für Sozialwissenschaften, S. 374-393.

Steiner, Gerhard (2006). Lernen und Wissenserwerb. In: Krapp, Andreas; Weidenmann, Bernd (Hrsg.), Pädagogische Psychologie: Ein Lehrbuch. Weinheim: Beltz PVU, S. 137202.

Tippelt, Rudolf; Mandl, Heinz; Straka, Gerald (2003). Entwicklung und Erfassung von Kompetenz in der Wissensgesellschaft - Bildungs- und wissenschaftstheoretische Perspektiven. In: Gogolin, Ingrid; Tippelt, Rudolf (Hrsg.), Innovation durch Bildung. 18. Kongress der deutschen Gesellschaft für Erziehungswissenschaft in München. Opladen: Leske + Budrich, S. 349-370.

Treumann, Klaus Peter (2005). Triangulation. In: Mikos, Lothar; Wegener, Claudia (Hrsg.), Qualitative Medienforschung: Ein Handbuch. Konstanz: UVK. S. 209-221.

Treumann, Klaus Peter; Meister, Dorothee M.; Sander, Uwe; Burkatzki, Eckhard; Hagedorn, Jörg, Kämmerer, Manuela; Strotmann, Mareike; Wegener, Claudia (Hrsg.) (2007). Medienhandeln Jugendlicher: Mediennutzung und Medienkompetenz. Bielefelder Medienkompetenzmodell. Wiesbaden: VS Verlag für Sozialwissenschaften.

Treumann, Klaus Peter; Arens, Markus; Ganguin, Sonja (2010). Die empirische Erfassung von Medienkompetenz mit Hilfe einer triangulativen Kombination qualitativer und quantitativer Forschungsmethoden. In: Harzig, Bodo; Meister, Dorothee; Moser, Heinz; Niesyto, Horst (Hrsg.), Jahrbuch Medienpädagogik 8. Medienkompetenz und Web 2.0. Wiesbaden: VS Verlag für Sozialwissenschaften. S. 163-182.

Tully, Claus J. (2004). Neue Lernkonzepte in der Informationsgesellschaft? - Eine Einführung. In: ders. (Hrsg.), Verändertes Lernen in modernen technisierten Welten. Organisierter und informeller Kompetenzerwerb Jugendlicher. Wiesbaden: VS Verlag für Sozialwissenschaften. S. 11-26.

Vollbrecht, Ralf (2003). Aufwachsen in Medienwelten. In: Fritz, Karsten u.a. (Hrsg.), Mediensozialisation: Pädagogische Perspektiven des Aufwachsens in Medienwelten. Opladen: Leske \& Budrich, S. 13-24.

Wagner, Bernd (2002). Kulturelle Globalisierung: Von Goethes «Weltliteratur» zu den weltweiten Teletubbies. In: Aus Politik und Zeitgeschichte, 12, S. 10-18.

Wiater, Werner (2003). Das Schulbuch als Gegenstand pädagogischer Forschung. In: ders. (Hrsg), Schulbuchforschung in Europa - Bestandsaufnahme und Zukunftsperspektive. Bad Heilbrunn: Klinkhardt. S.11-22.

Wild, Elke; Hofer, Manfred; Pekrun, Reinhard (2006). Psychologie des Lerners. In: Krapp, Andreas; Weidenmann, Bernd (Hrsg.), Pädagogische Psychologie: Ein Lehrbuch. Weinheim: Beltz PVU. S. 203-268. 